Published in final edited form as:

Curr Treat Options Oncol. 2016 June ; 17(6): 29. doi:10.1007/s11864-016-0405-5.

\title{
Is There a Role for Robotic Surgery in the Treatment of Head and Neck Cancer?
}

\author{
J. Kenneth Byrd, M.D. ${ }^{1}$ and Robert L. Ferris, M.D., Ph.D. ${ }^{2}$ \\ ${ }^{1}$ Department of Otolaryngology, Medical College of Georgia at Augusta University, 1120 15th \\ Street, BP 4109, Augusta, GA, 30912, USA \\ 2Department of Otolaryngology, University of Pittsburgh Medical Center, 200 Lothrop Street, EEI \\ 500, Pittsburgh, PA, 15213, USA
}

\section{Opinion statement}

Given the potential for long-term toxicities from concurrent chemoradiation, there is great interest in surgery as a primary treatment modality for head and neck cancers, particularly in the younger HPV-positive oropharyngeal cancer patient. Transoral robotic surgery (TORS) has proven to be an effective technique to safely treat oropharyngeal and select supraglottic tumors surgically. Sound, traditional surgical principles are employed using improved endoscopic visualization and precise instrumentation to perform oncologic surgery without the morbidity of transmandibular or transcervical approaches. Although level 1 evidence prospective clinical trials are currently underway for TORS, the literature supports its safety and efficacy based on numerous studies. Currently, prospective randomized trials are underway to provide better evidence for or against TORS in oropharyngeal cancer. Patient selection based on comorbidities, anatomy, and available pathological data is critical in choosing patients for TORS.

\section{Keywords}

Transoral robotic surgery; Oropharyngeal cancer; Head and neck cancer; TORS; Minimally invasive surgery

\section{Introduction}

Head and neck cancers, accounting for over 50,000 cases in the USA annually, present unique challenges to clinicians treating their patients due to the impact on function and quality of life of both the disease process and available treatments.

Traditional open approaches to the oropharynx for anything other than small tonsillar or palatal tumors were morbid, necessitating lateral pharyngotomy for small tumors [1] and

Correspondence to: Robert L. Ferris.

Compliance with Ethical Standards

Conflict of Interest

The authors declare that they have no conflict of interest.

Human and Animal Rights and Informed Consent

This article does not contain any studies with human or animal subjects performed by any of the authors. 
mandibulotomy or mandibulectomy [2, 3] for many tumors. For transmandibular approaches, specifically, cosmesis and function may suffer, particularly if mandibular nonunion occurs. Because of the equal oncologic control provided by nonoperative treatment with decrease serious complications [4], open surgery has largely been supplanted by radiation with or without chemotherapy since the results of the GORTEC trial and others [57].

The Food and Drug Administration (FDA) initially approved the Da Vinci robot (Sunnyvale, CA) for general surgical procedures in 2000. A master-slave design allows the surgeon to control four robotic arms from a console, usually located in the same operating room. Control of the robotic instrumentation mimics the natural motions of the surgeon, allowing a relatively steep learning curve to achieve proficiency [8]. A variety of articulating instruments, 5-8 mm, are available with precise articulation and high-definition optics via an endoscope. In general, thoracic, urologic, and GYN procedures, the robotic instruments are inserted into the abdominal or thoracic cavity via ports and controlled. However, the oral cavity provides a natural orifice for the introduction of robotic instruments to allow for surgical dissection in the upper aerodigestive tract.

After several IRB-approved prospective cohort studies, the FDA approved transoral robotic surgery (TORS) for early stage benign and malignant tumors of the upper aerodigestive tract in 2009 [9-14]. Although a variety of uses for robotic surgery in the head and neck have been reported in the literature, TORS is predominantly used for early stage oropharyngeal cancer and supraglottic cancer.

Critics argue that robotic surgery is expensive and superfluous and that patients should be treated with existing surgical and nonsurgical modalities. In this review, we will provide evidence that there is a role for TORS in management of head and neck neoplasms.

\section{Transoral robotic surgery: background}

The advent of transoral endoscopic techniques to approach the oropharynx has renewed interest in surgery as a primary treatment modality [15]. Transoral laser microsurgery (TLM) was initially popularized for early stage laryngeal cancers, but gained popularity in select regions for oropharyngeal carcinomas with control rates comparable to open surgery and nonoperative treatment [16]. In a nonrandomized, multicenter trial of 204 patients with oropharyngeal cancer, 3-year overall survival, disease specific survival, and disease-free survival were 86,88 , and $82 \%$, comparable to previous concurrent chemoradiation (CCRT) studies [17]. Advantages include excellent microscopic visualization of the tumor interface allowing sparing of normal tissue, tactile feedback during surgery, avoidance of tracheotomy and gastrostomy in most patients. Despite its reported success in experienced hands, the adoption of TLM for oropharyngeal surgery has been predominantly limited to a few highvolume centers. Because TLM often necessitates division of the specimen, in contrast to en bloc resection, some surgeons are adverse to the practice due to the potential for positive margins. 
The use of the Da Vinci robot for head and neck surgery was first reported in a porcine model of endoscopic submandibular gland removal and neck dissection in 2003 [18]. Weinstein and colleagues at the University of Pennsylvania recognized that a more practical use of robotic technology would be for transoral surgery and reported a canine study examining supraglottic laryngectomy in 2005 [19], followed by a human cadaveric and canine study of oropharyngeal surgery feasibility in 2006 [13]. After institutional review board approval, human trials for transoral robotic surgery (TORS) then began with a small patient series undergoing supraglottic partial laryngectomy successfully [10] and a larger group of patients who underwent robotic radical tonsillectomy [9].

Many other groups began to investigate robotic surgery of the head and neck at the same time, culminating with an early multicenter study to establish the safety and feasibility of TORS in a cohort of 192 patients with benign and malignant tumors of the oral cavity, oropharynx, and larynx. This study reported $4.3 \%$ positive margins and $16 \%$ rate of serious adverse events. Gastrostomy dependence was reported in $5 \%$ of patients, and tracheotomy was performed initially in $12 \%$ of patients ( $2.3 \%$ at last follow-up). The authors concluded that TORS is safe and feasible [20••].

Zevallos et al. recently reported effectiveness of TORS in practice by early adopters via data from the National Cancer Database. Although the length of stay was increased from 3 to 5 days, and the positive margin rate was higher than reported in the multicenter study (20 versus $4.3 \%$ ), the 30 day mortality remained low at $0.8 \%$. Predictors of positive margins in this study included T2 tumors, low-volume centers, and community-based cancer centers. The study highlighted the importance of surgeon experience, patient selection, and multidisciplinary care [21].

\section{Surgical procedure}

In TORS, the surgeon positions, or "docks," the robot at the surgical site, inserting two dissecting arms and the camera arm through the oral cavity with a retractor in place.

Although nasotracheal intubation provides some additional space in the oral cavity, it is not always necessary. If orotracheal intubation is performed, the tube should be secured intraorally with a suture to the mandible to prevent accidental extubation.

The angles of the two dissecting robotic arms form an acute angle of $30-45^{\circ}$, with the endoscope positioned between them. This angle becomes more acute the deeper the site of surgery lies. The axis of rotation of each arm is placed near the oral commissure.

Commonly, a 5-mm grasping instrument is used in one arm and monopolor electrocautery on the other. Alternatively, a second grasper may be used to hold a laser fiber [14], or the harmonic scalpel attachment may be used, although there is no distal articulation for the instrument. The $0^{\circ}$ or $30^{\circ}$ endoscope is used depending on the location of the tumor and anatomical exposure.

The surgeon then sits at a remote console with binocular, high-definition eyepieces to control the actions of the robot. An assistant sits at the bedside to provide counter-traction, 
provide suction, and to assist with hemostasis as needed. Dissection proceeds in a similar fashion to traditional transoral or open techniques.

Unilateral or bilateral neck dissection may be performed concurrently, prior to TORS, or after TORS, depending on surgeon preference [22]. Ligation of distal branches of the external carotid artery may be performed to decrease the risk of significant postoperative bleeding [23].

\section{TORS for supraglottic cancer}

As previously described, TORS was first reported for supraglottic partial laryngectomy (SGPL) [19]. Since then, the technique has gained some popularity and has been reported successfully by several groups [24-28]. Park and colleagues found that robotic supraglottic laryngectomy was associated with shorter operating times, length of stay, time to decannulation, and resumption of oral diet compared to open SGPL, with equal oncologic outcomes [29]. Although TLM has widely been accepted as a technique for endoscopic partial laryngeal surgery, some proponents of TORS argue that the robotic approach is equally effective and faster due to freedom of motion and the monopolar cautery.

Compared to TLM, however, some cases are challenging due to anatomy and the increased working space required for TORS. The Feyh-Katzenbach retractor's laryngeal blade is effective in many, but not all patients for exposure of the supraglottis, and the freedom of motion for the Da Vinci arms is restricted more inferiorly in the airway, which may hinder tumor resection [24]. Ansarin et al. compared 10 early TLM cases to 10 early TORS cases, finding a higher rate of positive margins in the TORS group [30].

Tracheotomy and nasogastric/gastrostomy feeding practices vary in the literature and likely depend on surgeon preference, patient selection, and aggressiveness of swallowing therapy. According to a multicenter European study of 84 patients undergoing TORS supraglottic laryngectomy, decannulation was likely within 2 weeks when tracheotomy was performed, and $9.5 \%$ of patients required gastrostomy, which is similar to other series [25, 26, 31]. As in open partial laryngeal surgery and TLM, patient selection and early speech therapy are paramount for TORS laryngeal surgery.

\section{TORS for oropharyngeal cancer}

Due to the morbidity of open approaches, the primary driver for TORS is oropharyngeal cancer. Although radical tonsillectomy can be safely performed with or without microscopic magnification or loupes, line of sight limits visibility and proponents argue that visualization of parapharyngeal structures is improved with robotic technique [9]. The base of tongue, however, is relatively inaccessible in many cases without a laryngoscope or angled endoscope. Many surgeons find that surgery of the tongue base is the most useful application of TORS, since it provides a binocular, three-dimensional, HD view of the surgical field for safe dissection around neurovascular structures.

To date, no prospective trials have compared TORS with adjuvant therapy versus (chemo) radiation, although several are currently underway $[15,32]$. Varying results of functional and 
oncologic outcomes have been reported in small series of patients. A systematic review comparing TORS-based treatment versus organ preservation therapy was published in 2014, including 8 IMRT studies and 12 TORS studies [33]. The authors found that oncologic outcomes were similar at 2 years and that the main difference lay in adverse outcomes. A similar meta-analysis performed by Morisod and Simon concluded that 5-year overall survival and disease-specific survival were equivalent between strategies [34].

To date, the largest, multicenter study of 410 patients [35••] demonstrated $91.8 \%$ 2-year locoregional control and $94.5 \%$ disease-specific survival. This compares favorably with published IMRT outcomes for early T-stage OPSCC in the radiation oncology literature [3638]. Of patients with data available, $52.7 \%$ required adjuvant treatment, $31.4 \%$ receiving radiation alone, and $21.3 \%$ receiving adjuvant chemoradiation [35••].

As an extension of oropharyngeal transoral robotic surgery, TORS has been employed as a method to locate the primary tumor in occult primary carcinomas [39]. Given the rise in HPV-related oropharyngeal cancers, a higher percentage of patients with unknown primary SCC may be localized to the tonsils and base of the tongue. As noted by Motz et al., the incidence of unknown primary SCC is rising, and the majority are HPV-related [40]. Byrd and colleagues reported localizing 19 of 22 patients ( $86.4 \%$ ) with nonlocalizing physical exams and imaging using TORS [41]. Other studies have reported similar success [42-44], with the ultimate goal to either treat the unknown primary surgically as small oropharyngeal tumors, or to limit radiation dose to the nasopharynx, hypopharynx, and contralateral pharyngeal constrictors via tumor localization.

Some have called the cost-effectiveness of robotic surgery into question, both in general and in otolaryngology. One prominent letter to the New England Journal of Medicine projected that if commonly performed general surgical, thoracic, gynecologic, and urologic procedures in the United States were converted to robotic surgery, an additional $\$ 2.5$ billion in healthcare costs would be generated annually if amortization was included [45]. The authors cautioned that careful cost-benefit analysis should be employed before accepting robotic surgery widely as the standard of care.

To date, several studies have investigated the economic implications of TORS. According to an analysis of national discharge date from the Nationwide Inpatient Sample, TORS is associated with shorter hospital stay, decreased gastrostomy tube and tracheotomy placement, and decreased hospital-related costs compared to open surgery [46]. Although not specific to transoral robotic surgery, a 2012 study querying cost via hospital reimbursement at two Mayo Clinic hospitals found that definitive chemoradiation was significantly more costly than transoral surgery-based strategies with or without adjuvant (chemo) radiation. In this study, cost was estimated from all inpatient, outpatient, and pharmacy reimbursements from treatment-related charges within 90 days of surgery [47]. Although the study was nonrandomized and uses reimbursements as a proxy for cost, it loosely approximates the third party payor perspective employed in economic evaluation of healthcare [48]. 
In order to have meaningful dialogue about economics in healthcare, cost must be framed according to outcomes in cost-effectiveness analysis, the ultimate goal of which is to provide outcome data in quality-adjusted life-years (QALYs). Via standard gamble techniques, de Almeida et al. provided utility states for outcomes of TORS and IMRT-based treatment of oropharyngeal cancers elicited from 50 healthy subjects. They found that utilities for outcome states of TORS were significantly higher than for (chemo) radiation, suggesting that subjects preferred the quality of life after robotic surgery [49]. From these utilities, de Almeida et al. subsequently performed a cost-effectiveness analysis comparing TORS-based strategies to radiotherapy or chemoradiation using a Markov model and probabilistic sensitivity analysis. In this study, TORS-based strategies demonstrated a cost savings of $\$ 1366$ in United States dollars and an increase of 0.25 QALYs over a 10-year time horizon [50••]. Although based on a single institution's cost data, this study also suggests that surgical-based treatments are less expensive than radiation or chemoradiation. Ultimately cost-effectiveness analysis embedded into prospective, randomized clinical trials will provide more definitive evidence about both each strategy.

\section{Clinical trials}

In order to provide level 1 evidence for surgical treatment of oropharyngeal cancer, including TORS, several surgical trials have recently begun to determine its effectiveness [15].

The significance of extracapsular spread in OPSCC has been questioned recently, as retrospective studies have demonstrated that it does not carry prognostic significance, particularly in HPV-positive patients $[51 \bullet \bullet, 52]$. Since ECS was identified as a contributor to poor outcomes in two randomized, controlled trials and is an indication for postoperative chemoradiation [53], level 1 evidence is required to make changes in our current treatment paradigms. In 2013, the ADEPT (Adjuvant De-escalation, Extracapsular Spread, p16 positive, Transoral) multi-institutional was launched at Washinton University in St. Louis, Mayo Clinic Scottsdale, and UT Southwestern. In this trial, the aim is to determine the benefit of chemotherapy in patients with HPV-related OPSCC with extracapsular extension, using 2-year disease free survival and locoregional control as endpoints.

The ECOG 3311 trial is a national phase II randomized prospective trial examining transoral resection of HPV-positive oropharyngeal cancer, followed by adjuvant therapy as guided by pathological features. Following transoral resection (TORS or TLM) and neck dissection, low risk patients are observed, and high risk patients receive 66 Gy with weekly cisplatin. Intermediate risk patients, defined as $<1 \mathrm{~mm}$ extracapsular spread, 2-4 positive lymph nodes, or perineural/lymphovascular invasion, are randomized to 50 or $60 \mathrm{~Gy}$ postoperatively. An estimated 377 patients will need to be enrolled to achieve significance in the randomized intermediate group, the endpoint of which is 2-year progression free survival. As of 2015, over 50 centers were credentialed and participating in the trial [15].

A comparison of oncologic efficacy in surgical versus nonsurgical approaches to oropharyngeal cancer is the next step following ECOG 3311 as a phase III trial. Currently, the ORATOR trial, a phase II trial of patients with HPV-positive and HPV-negative cancers 
of the oropharynx, is underway comparing TORS-based treatment to radiation with or without chemotherapy. It is a single institution trial in London, Ontario, with a required enrollment of 68 patients, the primary endpoint of which is quality of life at 1 year [32]. Although the secondary endpoints include OS and PFS, the study is not powered sufficiently or of sufficient duration to make definitive conclusions about the oncologic effectiveness of TORS compared to radiation-based treatments.

The RTOG 1221 trial was designed for early T-stage, p16-negative OPSCC. However, the study was closed in February 2015 due to failure to enroll any patients after 15 months. Participating surgeons found that in the study population, patients were presenting with more advanced disease not amenable to transoral approaches.

\section{Discussion}

The thrust of transoral surgery, including TLM and TORS, has been to spare patients longterm side effects of CCRT, which have not been extensively studied in survivors more than 2 years from treatment. This is of particular concern for patients with HPV-positive oropharyngeal cancer, some of whom may be expected to live 20-30 years after treatment. According to pooled data from the RTOG 91-11, RTOG 97-03, and RTOG 99-14 trials, $35 \%$ of oropharyngeal cancer survivors had severe late laryngopharyngeal toxicity, defined as chronic grade 3 or 4 pharyngeal/toxicity, requirement of a feeding tube beyond 2 years, or treatment-related death within 3 years [54]. Population-based estimates of late dysphagia in CCRT survivors are over $60 \%$ and are greater than those treated with surgery, radiation, or surgery and radiation [55]. Long-term (>2 year) reports of swallowing function after TORS are not well studied, but current evidence suggests a low (0-7\%) rate of gastrostomy dependence and low rate of significant dysphagia [56]. As swallowing has been reported to be a functional priority in head and neck cancer survivors, impairments significantly affect patients' quality of life and merit close attention in ongoing trials [57].

Late onset dysphagia can also greatly affect quality of life in survivors and may continue to progress years after treatment. In a series of patients with severe dysphagia $>5$ years after treatment with radiation or chemoradiation, $66 \%$ were gastrostomy-dependent despite therapy, and $86 \%$ developed pneumonia [58]. Although the incidence of severe late dysphagia in survivors could not be estimated from the series and is presumed to be rare, the authors noted that severe late dysphagia was very disabling for patients and refractory to treatment. Indeed, surgical treatment of patients with pharyngeal and esophageal stenosis is often fraught with frustration and failure.

The toxicities of chemoradiation are well known to medical oncologists and radiation oncologists, who are currently exploring ways to deintensify treatment via altered fractionation, decreased radiation dose, and less toxic chemotherapy regimens. Thus, a prospective randomized phase II trial was designed to address this clinical issue. ECOGACRIN (ECOG-ACRIN) 3311 examines the role of transoral head and neck surgery in treatment deintensification for stage III/IV p16+ oropharyngeal cancer (OPC). Patients with lateralized, resectable T1-T2 OPC and N1-N2b with no matted nodes undergo transoral resection and neck dissection, followed by risk-based adjuvant therapy. Intermediate risk 
patients (negative margins, $\mathrm{N} 2 \pm$ extranodal extension (ENE) $\leq \mathrm{mm}$ ) are randomized to 50 versus 60 Gy radiation. It is worth noting that the ECOG 3311 trial (Fig. 1) successfully passed its data safety monitoring committee (DMSC) review without activating stopping rules for excess toxicity or futility. Thus, far into the study, approximately $55 \%$ of patients have been assigned to the intermediate risk arm, and $12 \%$ have gone on to observation [15]. Thus, around $67 \%$ of patients have avoided concurrent chemoradiation. Detailed patient reported outcomes (PRO), quality of life, and swallowing analysis are being performed.

In terms of credentialing of surgeons and quality assurance, we established credentialing criteria and ongoing quality assurance (QA) for transoral endoscopic head and neck surgeons wishing to accrue to E3311. Each surgeon attests to experience of $>20$ cases of transoral resection of OPC, using either transoral robotic or laser microsurgery. Surgeons then submit paired surgical pathology and operative reports for 10 transoral resections within the past 24 months. Nine experienced head and neck surgeons established criteria for approval, review case submissions, on-hold status, and ongoing QA. Positive margin(s) were permitted on only 1 of 10 cases during initial credentialing or while accruing to E3311. Since 2013, 107 surgeons applied for credentialing. After peer-review, 71 surgeons were credentialed for E3311 at 51 different centers, with 49 surgeons having accrued $>1$ and 31 having accrued $\geq 3$ patients (accrual per surgeon $1-24$, median $=4$ ). Twelve surgeons were asked to provide additional, replacement cases, where histology or margin status was unclear or deemed insufficient during review. Of these, 10 were approved upon re-review. Two surgeons withdrew their applications, and 22 surgeons have not formally submitted cases for credentialing review. Ongoing QA has led to review of two surgeons with positive margin(s) in their first 5 cases. The composite QA stopping rule of $22 \%$ combining grade III/IV bleeding and positive margins was not met during planned DSMC review (August 2015), with $8 \%$ grade III/IV bleed rate and $3 \%$ positive margins. Thus, initial surgeon credentialing and ongoing QA results in low rates of positive margins and grade III/IV oral bleeding rates in a multicenter trial of transoral surgery.

In the literature outside of a clinical trial, a higher proportion of patients routinely receive chemoradiation after TORS [33]. Potentially, extranodal extension (ENE) in lymph nodes may account for a significant number of these patients, which may be decreased in the future pending the outcome of trials investigating it as a prognostic feature. An additional factor, however, is likely patient selection and surgeon experience related to the learning curve and patient volume. In the ECOG 3311 trial, surgeons must undergo credentialing to participate; therefore, the trial has selected experienced, high-volume participants who likely have better outcomes than low-volume surgeons or new adopters. In order to ensure that the ultimate findings of the trial persist in widespread application, proper training and multidisciplinary input for patient selection are paramount.

Nevertheless, until indications for postoperative chemotherapy are formally changed within our practice guidelines, a significant proportion of patients may continue to require postoperative chemotherapy due to ECS spread found during neck dissection, as it cannot be reliably predicted in all patients based on preoperative imaging [59]. The incidence of ECS in OPSCC is $23 \%$ in surgically treated patients according to the SEER database and increases with $\mathrm{N}$-staging [51••]; however, the actual percentage may be found to be higher as 
additional patients who previously would have undergone (chemo) radiation are selected for surgery. It is important to note, however, that surgical treatment of the primary and neck provides valuable pathologic staging to guide treatment, which may be particularly useful in HPV-negative patients whose outcomes are less favorable overall. Additionally, although avoidance of triple therapy is the goal in patient selection, a percentage of patients undergoing IMRT-based strategies also require salvage neck dissection [33] or salvage of the primary, and so employing surgery, radiation, and chemotherapy to some degree are unavoidable.

In our practice, several factors are employed in selection for TORS-based treatment in supraglottic and oropharyngeal cancer, including patient preference and overall health. Each case should be presented in a multidisciplinary setting with input from medical oncology and radiation oncology, and patients should be counseled about the risks and benefits of both approaches when dual candidacy is present. Preoperative evaluation by a speech and language pathologist is helpful to determine if any underlying dysphagia is present that may significantly worsen recovery.

Specifically for supraglottic cancer, heavy nodal burden necessitating bilateral neck radiation postoperatively is a relative contraindication, as IMRT fields will have some degree of overlap with the pharyngeal constrictors and potentially worsen swallowing outcomes. Preoperative pulmonary function and swallowing are also important, as in TLM, since patients will need to temporarily tolerate some degree of penetration/aspiration in the postoperative period. Anatomically, if the tumor has significant infrahyoid or glottis extension, TLM may be a more appropriate technique due to the limited working space and thermal spread of the monopolar cautery. In preparation for surgery, we recommend having a laryngoscope and $\mathrm{CO}_{2}$ laser available when TORS SGPL is planned, in the event that exposure is inadequate.

For patients with OPSCC, relative contraindications in our practice are obvious extracapsular extension on imaging, significant palatal involvement, encroachment into the vallecula that would necessitate supraglottic resection and T4 tumors. Patients are presented with the risk of postoperative chemoradiation based on available evidence. Patients with preoperative dysphagia who have tongue base tumors approaching or crossing midline may have better swallowing outcomes with nonoperative treatment, as well.

In patients who require salvage TORS after chemoradiation, additional considerations include airway edema postoperative hemorrhage [23], the potential for fistula, velopharyngeal insufficiency, and carotid exposure. In salvage TORS SGPL, airway edema and dysphagia are a major concern, and tracheotomy and gastrostomy are more often needed than in primary TORS. Salvage TORS has been reported successfully with an acceptable complication rate without routine reconstruction [60]. In our practice, local flap or free tissue transfer is employed to minimize the chance for carotid blowout, restore tongue base bulk, and improve velopharyngeal closure in salvage oropharyngectomy, as radiation changes decrease the amount of parapharyngeal fat and hinder mobility via fibrosis [61]. 
In summary, TORS is a valuable adjunct to open techniques and transoral laser surgery that allows appropriately selected patients to be treated surgically. Retrospective evidence demonstrates oncologic outcomes equal to traditional techniques and nonoperative treatment with a possible benefit to long-term quality of life. Currently, prospective, randomized trials are underway to provide more definitive evidence for or against transoral surgery.

\section{References and Recommended Reading}

Papers of particular interest, published recently, have been highlighted as:

•• Of major importance

1. Stern SJ. Anatomy of the lateral pharyngotomy approach. Head Neck. 1992; 14(2):153-6. [PubMed: 1601654]

2. Shaha AR. Mandibulotomy and mandibulectomy in difficult tumors of the base of the tongue and oropharynx. Semin Surg Oncol. 1991; 7(1):25-30. [PubMed: 2003182]

3. Spiro RH, Gerold FP, Shah JP, Sessions RB, Strong EW. Mandibulotomy approach to oropharyngeal tumors. Am J Surg. 1985; 150(4):466-9. [PubMed: 4051109]

4. Parsons JT, Mendenhall WM, Stringer SP, et al. Squamous cell carcinoma of the oropharynx: surgery, radiation therapy, or both. Cancer. 2002; 94(11):2967-80. [PubMed: 12115386]

5. Calais G, Alfonsi M, Bardet E, et al. Randomized trial of radiation therapy versus concomitant chemotherapy and radiation therapy for advanced-stage oropharynx carcinoma. J Natl Cancer Inst. 1999; 91(24):2081-6.

6. Ang KK, Harris J, Wheeler R, et al. Human papillomavirus and survival of patients with oropharyngeal cancer. N Engl J Med. 2010; 363(1):24-35. [PubMed: 20530316]

7. Pignon JP, le Maitre A, Maillard E, Bourhis J, Group M-NC. Meta-analysis of chemotherapy in head and neck cancer (MACH-NC): an update on 93 randomised trials and 17,346 patients. Radiother Oncol J Eur Soc Ther Radiol Oncol. 2009; 92(1):4-14.

8. White HN, Frederick J, Zimmerman T, Carroll WR, Magnuson JS. Learning curve for transoral robotic surgery: a 4-year analysis. JAMA Otolaryngol Head Neck Surg. 2013; 139(6):564-7. [PubMed: 23680949]

9. Weinstein GS, O’Malley BW Jr, Snyder W, Sherman E, Quon H. Transoral robotic surgery: radical tonsillectomy. Arch Otolaryngol Head Neck Surg. 2007; 133(12):1220-6. [PubMed: 18086963]

10. Weinstein GS, O’Malley BW Jr, Snyder W, Hockstein NG. Transoral robotic surgery: supraglottic partial laryngectomy. Ann Otol Rhinol Laryngol. 2007; 116(1):19-23. [PubMed: 17305273]

11. Boudreaux BA, Rosenthal EL, Magnuson JS, et al. Robot-assisted surgery for upper aerodigestive tract neoplasms. Arch Otolaryngol Head Neck Surg. 2009; 135(4):397-401. [PubMed: 19380364]

12. Genden EM, Desai S, Sung CK. Transoral robotic surgery for the management of head and neck cancer: a preliminary experience. Head Neck. 2009; 31(3):283-9. [PubMed: 18972413]

13. O’Malley BW Jr, Weinstein GS, Snyder W, Hockstein NG. Transoral robotic surgery (TORS) for base of tongue neoplasms. Laryngoscope. 2006; 116(8):1465-72. [PubMed: 16885755]

14. Solares CA, Strome M. Transoral robot-assisted CO2 laser supraglottic laryngectomy: experimental and clinical data. Laryngoscope. 2007; 117(5):817-20. [PubMed: 17473675]

15. Holsinger FC, Ferris RL. Transoral endoscopic head and neck surgery and its role within the multidisciplinary treatment paradigm of oropharynx cancer: robotics, lasers, and clinical trials. J Clin Oncol Off J Am Soc Clin Oncol. 2015; 33(29):3285-92.

16. Rich JT, Milov S, Lewis JS Jr, Thorstad WL, Adkins DR, Haughey BH. Transoral laser microsurgery (TLM) +/- adjuvant therapy for advanced stage oropharyngeal cancer: outcomes and prognostic factors. Laryngoscope. 2009; 119(9):1709-19. [PubMed: 19572271]

17. Haughey BH, Hinni ML, Salassa JR, et al. Transoral laser microsurgery as primary treatment for advanced-stage oropharyngeal cancer: a United States multicenter study. Head Neck. 2011; 33(12):1683-94. [PubMed: 21284056] 
18. Haus BM, Kambham N, Le D, Moll FM, Gourin C, Terris DJ. Surgical robotic applications in otolaryngology. Laryngoscope. 2003; 113(7):1139-44. [PubMed: 12838011]

19. Weinstein GS, O’Malley BW Jr, Hockstein NG. Transoral robotic surgery: supraglottic laryngectomy in a canine model. Laryngoscope. 2005; 115(7):1315-9. [PubMed: 15995528]

20••. Weinstein GS, O’Malley BW Jr, Magnuson JS, et al. Transoral robotic surgery: a multicenter study to assess feasibility, safety, and surgical margins. Laryngoscope. 2012; 122(8):1701-7. Multicenter study establishing safety and feasibility of transoral robotic surgery. [PubMed: 22752997]

21. Zevallos JP, Mitra N, Swisher-McClure S. Patterns of care and perioperative outcomes in transoral endoscopic surgery for oropharyngeal squamous cell carcinoma. Head Neck. 2016; 38(3):402-9. [PubMed: 25351184]

22. Kucur C, Durmus K, Gun R, et al. Safety and efficacy of concurrent neck dissection and transoral robotic surgery. Head Neck. 2015

23. Mandal R, Duvvuri U, Ferris RL, Kaffenberger TM, Choby GW, Kim S. Analysis of post-transoral robotic-assisted surgery hemorrhage: frequency, outcomes, and prevention. Head Neck. 2015

24. Olsen SM, Moore EJ, Koch CA, Price DL, Kasperbauer JL, Olsen KD. Transoral robotic surgery for supraglottic squamous cell carcinoma. Am J Otolaryngol. 2012; 33(4):379-84. [PubMed: 22133967]

25. Ozer E, Alvarez B, Kakarala K, Durmus K, Teknos TN, Carrau RL. Clinical outcomes of transoral robotic supraglottic laryngectomy. Head Neck. 2013; 35(8):1158-61. [PubMed: 22907898]

26. Lallemant B, Chambon G, Garrel R, et al. Transoral robotic surgery for the treatment of T1-T2 carcinoma of the larynx: preliminary study. Laryngoscope. 2013; 123(10):2485-90. [PubMed: 23918439]

27. Park YM, Kim WS, Byeon HK, Lee SY, Kim SH. Surgical techniques and treatment outcomes of transoral robotic supraglottic partial laryngectomy. Laryngoscope. 2013; 123(3):670-7. [PubMed: 23288629]

28. Mendelsohn AH, Remacle M, Van Der Vorst S, Bachy V, Lawson G. Outcomes following transoral robotic surgery: supraglottic laryngectomy. Laryngoscope. 2013; 123(1):208-14. [PubMed: 23008093]

29. Park YM, Byeon HK, Chung HP, Choi EC, Kim SH. Comparison of treatment outcomes after transoral robotic surgery and supraglottic partial laryngectomy: our experience with seventeen and seventeen patients respectively. Clin Otolaryngol Off J ENT-UK Off J Netherlands Soc Oto-RhinoLaryngol Cervico-Facial Surg. 2013; 38(3):270-4.

30. Ansarin M, Zorzi S, Massaro MA, et al. Transoral robotic surgery vs transoral laser microsurgery for resection of supraglottic cancer: a pilot surgery. Int J Med Robot Comput Assisted Surg MRCAS. 2013

31. Razafindranaly V, Lallemant B, Aubry K, et al. Clinical outcomes with transoral robotic surgery for supraglottic squamous cell carcinoma: experience of a French evaluation cooperative subgroup of GETTEC. Head Neck. 2015

32. Nichols AC, Yoo J, Hammond JA, et al. Early-stage squamous cell carcinoma of the oropharynx: radiotherapy vs. trans-oral robotic surgery (ORATOR)—study protocol for a randomized phase II trial. BMC Cancer. 2013; 13:133. [PubMed: 23514246]

33. de Almeida JR, Byrd JK, Wu R, et al. A systematic review of transoral robotic surgery and radiotherapy for early oropharynx cancer: a systematic review. Laryngoscope. 2014; 124(9):2096102. [PubMed: 24729006]

34. Morisod B, Simon C. Meta-analysis on survival of patients treated with transoral surgery versus radiotherapy for early-stage squamous cell carcinoma of the oropharynx. Head Neck. 2014

35••. de Almeida JR, Li R, Magnuson JS, et al. Oncologic outcomes after transoral robotic surgery: a multi-institutional study. JAMA Otolaryngol Head Neck Surg. 2015; 141(12):1043-51. The largest prospective study to date demonstrating oncologic efficacy of transoral robotic surgery for oropharyngeal cancer. [PubMed: 26402479]

36. Mendenhall WM, Amdur RJ, Morris CG, Kirwan JM, Li JG. Intensity-modulated radiotherapy for oropharyngeal squamous cell carcinoma. Laryngoscope. 2010; 120(11):2218-22. [PubMed: 20938964] 
37. Eisbruch A, Harris J, Garden AS, et al. Multi-institutional trial of accelerated hypofractionated intensity-modulated radiation therapy for early-stage oropharyngeal cancer (RTOG 00-22). Int J Radiat Oncol Biol Phys. 2010; 76(5):1333-8. [PubMed: 19540060]

38. Garden AS, Morrison WH, Wong PF, et al. Disease-control rates following intensity-modulated radiation therapy for small primary oropharyngeal carcinoma. Int J Radiat Oncol Biol Phys. 2007; 67(2):438-44. [PubMed: 17141972]

39. Mehta V, Johnson P, Tassler A, et al. A new paradigm for the diagnosis and management of unknown primary tumors of the head and neck: a role for transoral robotic surgery. Laryngoscope. 2013; 123(1):146-51. [PubMed: 23154813]

40. Motz K, Qualliotine JR, Rettig E, Richmon JD, Eisele DW, Fakhry C. Changes in unknown primary squamous cell carcinoma of the head and neck at initial presentation in the era of human papillomavirus. JAMA Otolaryngol Head Neck Surg. 2016:1-7.

41. Byrd JK, Smith KJ, de Almeida JR, et al. Transoral robotic surgery and the unknown primary: a cost-effectiveness analysis. Otolaryngol Head Neck Surg Off J Am Acad Otolaryngol Head Neck Surg. 2014; 150(6):976-82.

42. Durmus K, Rangarajan SV, Old MO, Agrawal A, Teknos TN, Ozer E. Transoral robotic approach to carcinoma of unknown primary. Head Neck. 2013

43. Kang SY, Dziegielewski PT, Old MO, Ozer E. Transoral robotic surgery for carcinoma of unknown primary in the head and neck. J Surg Oncol. 2015; 112(7):697-701. [PubMed: 26437845]

44. Patel SA, Magnuson JS, Holsinger FC, et al. Robotic surgery for primary head and neck squamous cell carcinoma of unknown site. JAMA Otolaryngol Head Neck Surg. 2013; 139(11):1203-11. [PubMed: 24136446]

45. Barbash GI, Glied SA. New technology and health care costs - the case of robot-assisted surgery. N Engl J Med. 2010; 363(8):701-4. [PubMed: 20818872]

46. Richmon JD, Quon H, Gourin CG. The effect of transoral robotic surgery on short-term outcomes and cost of care after oropharyngeal cancer surgery. Laryngoscope. 2014; 124(1):165-71. [PubMed: 23945993]

47. Moore EJ, Hinni ML, Olsen KD, Price DL, Laborde RR, Inman JC. Cost considerations in the treatment of oropharyngeal squamous cell carcinoma. Otolaryngol Head Neck Surg Off J Am Acad Otolaryngol Head Neck Surg. 2012; 146(6):946-51.

48. Gold M. Panel on cost-effectiveness in health and medicine. Med Care. 1996; 34(12 Suppl):DS197-9. [PubMed: 8969326]

49. de Almeida JR, Villanueva NL, Moskowitz AJ, et al. Preferences and utilities for health states after treatment for oropharyngeal cancer: transoral robotic surgery versus definitive (chemo) radiotherapy. Head Neck. 2014; 36(7):923-33. [PubMed: 23595774]

50••. de Almeida JR, Moskowitz AJ, Miles BA, et al. Cost-effectiveness of transoral robotic surgery versus (chemo) radiotherapy for early $\mathrm{T}$ classification oropharyngeal carcinoma: a cost-utility analysis. Head Neck. 2014; 38(4):589-600. Cost-effectiveness analysis demonstrating that surgical based strategies for oropharyngeal cancer are less costly and associated with increased quality-adjusted life years compared to nonsurgical treatment.

51••. Maxwell JH, Ferris RL, Gooding W, et al. Extracapsular spread in head and neck carcinoma: impact of site and human papillomavirus status. Cancer. 2013; 119(18):3302-8. Retrospective study demonstrating that extracapsular extension does not carry the same prognosis for oropharyngeal cancers. [PubMed: 23797868]

52. Sinha P, Lewis JS Jr, Piccirillo JF, Kallogjeri D, Haughey BH. Extracapsular spread and adjuvant therapy in human papillomavirus-related, p16-positive oropharyngeal carcinoma. Cancer. 2012; 118(14):3519-30. [PubMed: 22086669]

53. Bernier J, Cooper JS, Pajak TF, et al. Defining risk levels in locally advanced head and neck cancers: a comparative analysis of concurrent postoperative radiation plus chemotherapy trials of the EORTC (\#22931) and RTOG (\# 9501). Head Neck. 2005; 27(10):843-50. [PubMed: 16161069]

54. Machtay M, Moughan J, Trotti A, et al. Factors associated with severe late toxicity after concurrent chemoradiation for locally advanced head and neck cancer: an RTOG analysis. J Clin Oncol Off J Am Soc Clin Oncol. 2008; 26(21):3582-9. 
55. Francis DO, Weymuller EA Jr, Parvathaneni U, Merati AL, Yueh B. Dysphagia, stricture, and pneumonia in head and neck cancer patients: does treatment modality matter? Ann Otol Rhinol Laryngol. 2010; 119(6):391-7. [PubMed: 20583737]

56. Hutcheson KA, Holsinger FC, Kupferman ME, Lewin JS. Functional outcomes after TORS for oropharyngeal cancer: a systematic review. Eur Arch Oto Rhino Laryngol Off J Eur Fed Otorhinolaryngol Soc (EUFOS): Affiliated German Soc Otorhinolaryngol Head Neck Surg. 2015; 272(2):463-71.

57. Wilson JA, Carding PN, Patterson JM. Dysphagia after nonsurgical head and neck cancer treatment: patients' perspectives. Otolaryngol Head Neck Surg Off J Am Acad Otolaryngol Head Neck Surg. 2011; 145(5):767-71.

58. Hutcheson KA, Lewin JS, Barringer DA, et al. Late dysphagia after radiotherapy-based treatment of head and neck cancer. Cancer. 2012; 118(23):5793-9. [PubMed: 23640737]

59. Maxwell JH, Rath TJ, Byrd JK, et al. Accuracy of computed tomography to predict extracapsular spread in p16-positive squamous cell carcinoma. Laryngoscope. 2015; 125(7):1613-8. [PubMed: 25946149]

60. White H, Ford S, Bush B, et al. Salvage surgery for recurrent cancers of the oropharynx: comparing TORS with standard open surgical approaches. JAMA Otolaryngol Head Neck Surg. 2013; 139(8):773-8. [PubMed: 23949352]

61. de Almeida JR, Park RC, Genden EM. Reconstruction of transoral robotic surgery defects: principles and techniques. J Reconstr Microsurg. 2012; 28(7):465-72. [PubMed: 22744899] 


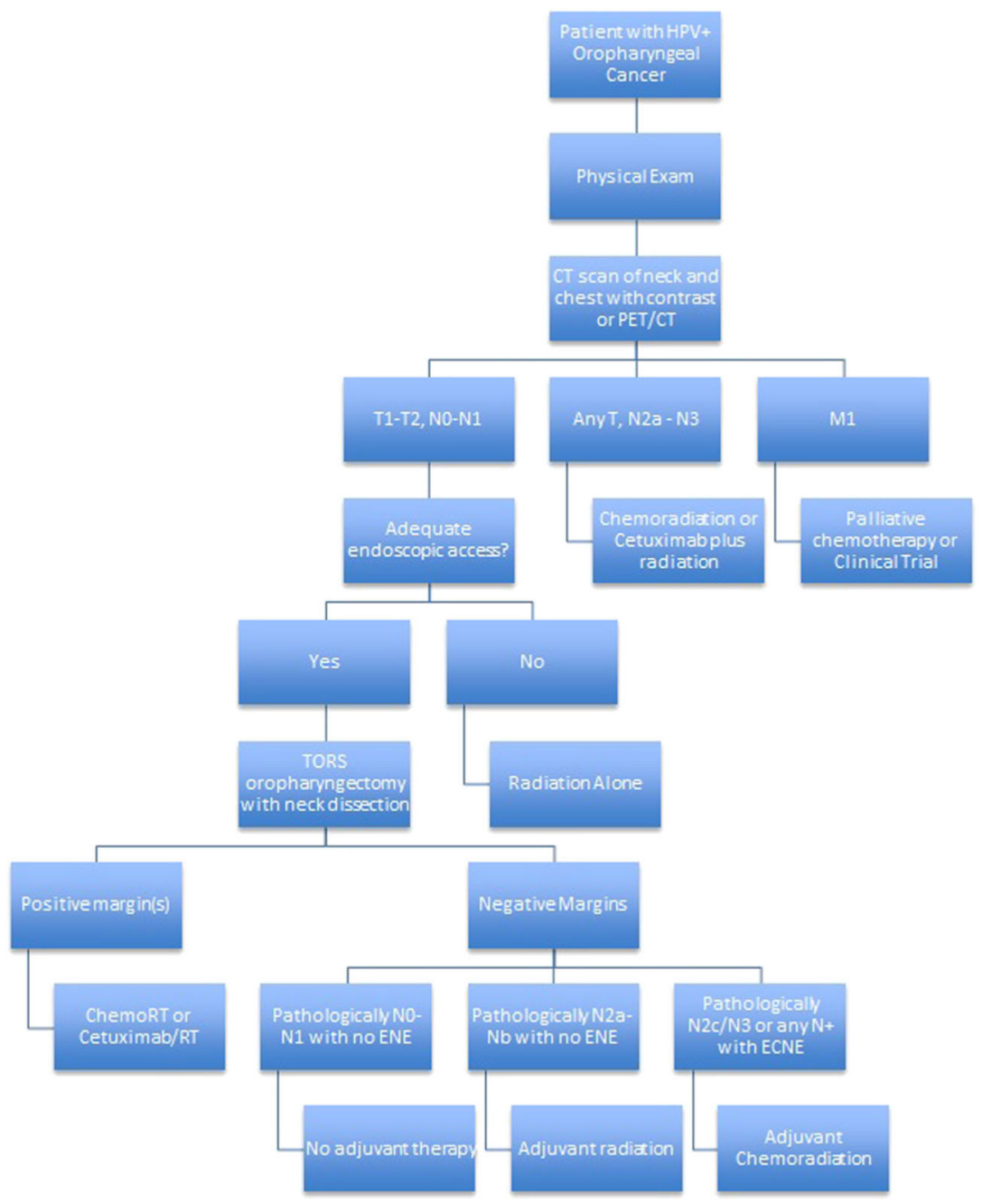

Fig. 1.

Schema for ECOG 3311 phase II randomized clinical trial of transoral surgery for HPVrelated oropharyngeal cancer. 